\title{
Jamming and percolation of dimers in restricted-valence random sequential adsorption
}

\author{
A. P. Furlan $\oplus^{*}$ \\ Departamento de Física, ICEx, Universidade Federal de Minas Gerais, C. P. 702, 30123-970 Belo Horizonte, Minas Gerais, Brazil \\ Diogo C. dos Santos $\odot$ \\ Departamento de Estatística, ICEx, Universidade Federal de Minas Gerais, 30123-970 Belo Horizonte, Minas Gerais, Brazil \\ Robert M. Ziff \\ Center for the Study of Complex Systems and Department of Chemical Engineering, University of Michigan, \\ Ann Arbor, Michigan 48109-2136, USA \\ Ronald Dickman (1) \\ Departamento de Física and National Institute of Science and Technology for Complex Systems, ICEx, Universidade Federal de Minas \\ Gerais, C. P. 702, 30123-970 Belo Horizonte, Minas Gerais, Brazil
}

(Received 15 June 2020; accepted 1 September 2020; published 5 October 2020)

\begin{abstract}
Restricted-valence random sequential adsorption is studied in its pure and disordered versions on the square and triangular lattices. For the simplest case (pure on the square lattice) we prove the absence of percolation for maximum valence $V_{\max }=2$. In other cases, Monte Carlo simulations are used to investigate the percolation threshold, universality class, and jamming limit. Our results reveal a continuous transition for the majority of the cases studied. The percolation threshold is computed through finite-size scaling analysis of seven properties; its value increases with the average valency. Scaling plots and data-collapse analyses show that the transition belongs to the standard percolation universality class even in disordered cases.
\end{abstract}

DOI: 10.1103/PhysRevResearch.2.043027

\section{INTRODUCTION}

Percolation [1] is characterized by the formation of a spanning cluster in a system composed of elements (sites and/or bonds), each present independently with probability $p$. The probability of a spanning or percolating cluster is only nonzero for $p>p_{c}$, the percolation threshold, marking a continuous phase transition with associated critical exponents [2-4]. Percolation has found a huge variety of applications, such as granular materials [5], forest fires [6], polymers [7-10], porous media [11,12], and biological evolution [13].

Random sequential adsorption (RSA) [2,14] is a stochastic process consisting in irreversible deposition of immobile objects onto an initially empty substrate such that each object excludes a certain area from further occupation. A realization of RSA stops when no further deposition events are possible, at which point the system is said to be jammed. Introducing a deposition attempt rate (per unit area, or per site, on a lattice) of unity, a time can be associated with each deposition event in a given realization. Letting the coverage $\rho$ denote

\footnotetext{
*apfurlan@ fisica.ufmg.br

Published by the American Physical Society under the terms of the Creative Commons Attribution 4.0 International license. Further distribution of this work must maintain attribution to the author(s) and the published article's title, journal citation, and DOI.
}

the fraction of the substrate occupied by deposited objects, we define $\rho(t)$ as the mean coverage (over all realizations) at time $t$. Of particular interest is the jamming or saturation coverage $\rho_{\infty} \equiv \lim _{t \rightarrow \infty} \rho(t)$. RSA of objects of diverse formats (disks [15], linear $k$-mers [16,17], ellipsoids [18], tetrahedral particles [19], etc.) has been used to model a wide range of physical processes such as ion implantation in semiconductors [20], protein adsorption [2], as well as the original car-parking problem [21].

Consider RSA on a two-dimensional lattice, of objects occupying two or more sites. As the coverage increases, it may be possible for the set of deposited objects to "percolate," i.e., to form a spanning cluster, in which case the mean coverage at percolation is a quantity of interest. (While RSA of monomers - objects occupying a single site - is trivial, percolation of monomers is a classic problem, and $p_{c}$ has been determined, exactly or numerically, for a wide variety of lattices [22-27].) RSA of extended objects, such as rods or linear lattice $k$-mers, is a problem of current interest. Cherkasova et al. [28] showed that the percolation threshold for dimers ( $k$-mers with $k=2$ ) is smaller than for monomers. As expected, the universality class does not depend on the length $k$. In addition, these authors observed that when the dimers can only align along one direction, the percolation threshold increases.

Cornette et al. [29,30] studied the influence of $k$ in the percolation threshold via Monte Carlo simulation (MC) and Bethe lattice analysis. They confirmed that $p_{c}$ decreases 


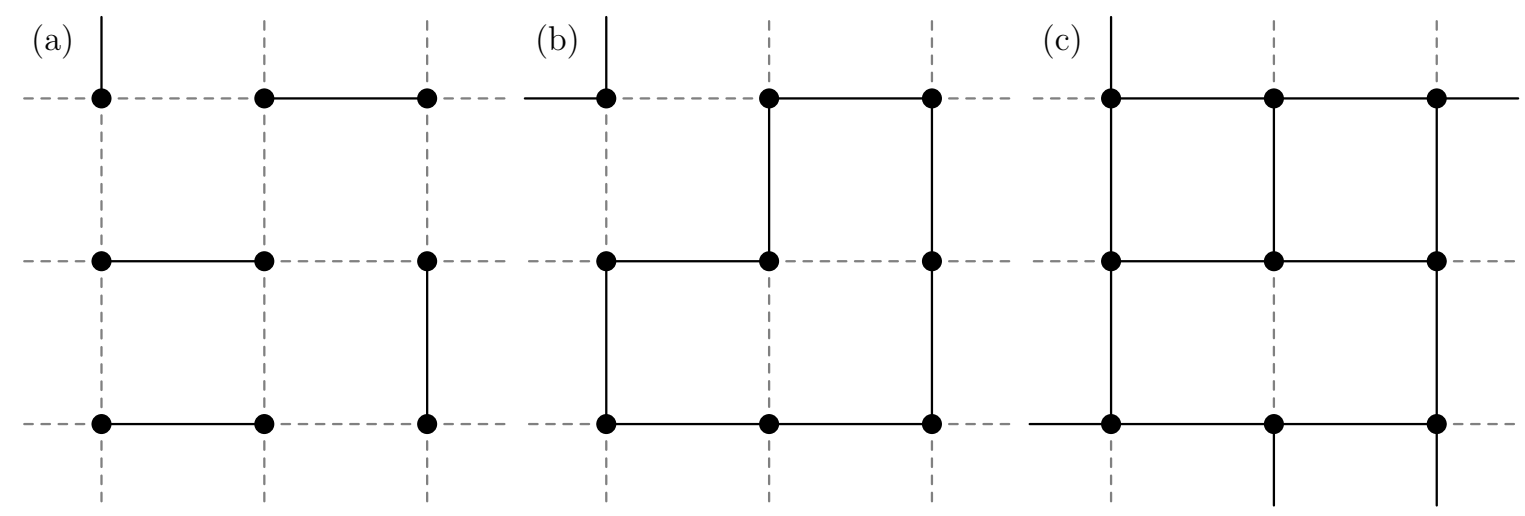

FIG. 1. Solid lines, dashed lines, and circles represent occupied edges, unoccupied edges, and occupied sites, respectively. Panels (a), (b), and (c) show possible configurations of systems with $V_{\max }=1,2$, and 3, respectively.

monotonically with $k$ for $1 \leqslant k \leqslant 16$ and that the universality class of the transition is standard percolation, independent of $k$. Leroyer and Pommiers [31] demonstrated that the percolation threshold $p_{c}$ decreases with increasing segment length, reaches a minimum value, and then increases for $k \geqslant 15$. Tarasevich et al. $[32,33]$ conjectured, based on simulation, that for $k$-mers of sufficient length $\left(k \gtrsim 1.2 \times 10^{4}\right)$ percolation does not occur. More recently, Kondrat et al. [34] developed a rigorous proof refuting this conjecture. They showed that for nonoverlapping $k$-mers, the jammed configuration includes a percolating cluster. This assertion was confirmed by Slutskii et al. [26] in an extensive computational study.

Although deposition of particles or $k$-mers on a regular substrate has attracted much attention, this model needs to be extended to describe more realistic situations. In many systems, the substrate includes impurities and/or defects that affect the deposition process. A simple realization of substrate disorder involves randomly excluding a fraction of sites from the deposition process, resulting in a diluted system. For example, Cornette et al. [35] studied deposition of polyatomic structures on diluted lattices, observing that the percolation threshold increases with dilution and that for dilutions greater than a certain value, there is no percolation $[33,35]$. Following a similar line, Lebovka et al. [36] considered a RSA process with defects on the substrate as well as on the $k$-mers. They observed that the defects hinder the percolation process even at low concentration and that for sufficiently large values of $k$, percolation does not occur.

In standard dimer RSA, a given site can be occupied by at most one monomer, and only one dimer can be incident upon an occupied site. A way of softening this restriction, while still prohibiting arbitrarily high densities is to allow up to $V_{\max }$ dimers to be incident on a given site. In the resulting restricted-valence RSA process, each lattice edge can be occupied by at most one dimer. Here, $V_{\max }$ ranges from unity (the usual dimer RSA problem) up to $q$, the lattice coordination number. (In the latter case, the RSA process is trivial since all edges are eventually occupied.) Restricted-valence RSA is analogous to a self-avoiding random walk (SAW) in which the walk is allowed to visit the same site up to $K$ times. Krawczyk et al. [37] showed that the existence and nature of phase transitions in these models depends on the details and dimensionality. For example, in case of forbidden reversal on the cubic lattice, discontinuous and continuous transitions are observed, with the continuous transition of the same type as in interacting self-avoiding walk collapse (ISAW) [38]. On the other hand, for allowed reversal on the square lattice, these authors do not find any indication of a phase transition. Oliveira et al. [39] studied SAWs with $K=2$ using Husimi and Bethe lattice solutions. They found a rich phase diagram with regular polymerized, nonpolymerized, and pair-polymerized stable phases, a tricritical point, and a critical endpoint. The transition between polymerized and nonpolymerized phases can be continuous or discontinuous, depending on the region of the phase diagram.

We consider restricted-valence RSA (and the associated percolation problem) on the square and triangle lattices, in both their pure form (all sites having the same maximum valence) and with disorder, such that the maximum valences at each site are independent, identically distributed random variables. Our objective is to understand how percolation thresholds and jamming limits depend on the valence restrictions. We verify that percolation transitions, when they exist, belong to the standard percolation universality class. In addition, we develop a proof for the absence of percolation for a maximum valence of 2 .

The remainder of this paper is organized as follows. In Sec. II we detail the restricted-valence RSA model. Simulation methods are presented in Sec. III, followed by results in Sec. IV. Finally, in Sec. V we present our conclusions. The proof is given in the Appendix.

\section{MODEL}

We study RSA of dimers on a regular lattice under the restriction that the number of dimers that can attach to a vertex (its valence) cannot exceed $V_{\max }$. In case $V_{\max }=q$, with $q$ the coordination number of the lattice, there is no restriction and all edges of the lattice are eventually covered by a dimer. The cases $V_{\max }=1,2$, and 3 on the square lattice are shown in Figs. 1(a), 1(b) and 1(c), respectively. For $V_{\max }=1$, we have the usual irreversible dimer or domino tiling problem [40], in which only isolated edges may be occupied [see Fig. 1(a)]. For $V_{\max }=2$, a vertex may have a maximum of two incident edges, giving rise to open or closed nonbranching paths, as shown in Fig. 1(b). Figure 1(c) shows a typical configuration 
for $V_{\max }=3$. In Fig. 1 the bonds denoted by dashed lines cannot be occupied, as this would violate the maximum-valence condition. Vertices with valence $V_{\max }$ are said to be saturated.

The RSA process is conveniently represented by associating times $t_{i}$ to each edge $i$ of the lattice. The $t_{i}$, which are chosen anew at each realization of the process, are independent, identically distributed random variables, uniform on $(0,1]$. At time zero the lattice is empty (all edges unoccupied). At time $t_{\min } \equiv \min _{i}\left\{t_{i}\right\}$ the edge corresponding to $t_{\min }$ becomes occupied. Subsequently, edges are visited according to their associated times and occupied if this does not violate the maximum-valence conditions. Occupation is irreversible. Thus an unoccupied edge with one or more saturated vertices can never be occupied. Although percolating configurations exist for $V_{\max }=2$, the percolation probability is zero in this case, as shown in the Appendix.

In simulations on a periodic lattice of $N$ sites, there are $N_{\mathrm{e}}=N q / 2$ edges. The edges are ranked in order of increasing time and occupied (if permitted by the maximum-valence restrictions) in that order. We associate a discrete time $p_{i}=$ $n_{i} / N_{\mathrm{e}}$ with edge $i$, where $n_{i} \in\left\{1, \ldots, N_{\mathrm{e}}\right\}$ is the position of edge $i$ on the ordered list. Thus for discrete time $p=0.5$, half the edges have been visited in the RSA process. The process terminates when the system is jammed, which happens for some (sample-dependent) $p_{\text {final }} \leqslant 1$.

\section{SIMULATION}

We use a variation of the union-find algorithm of Newman and Ziff $[41,42]$ (NZ) to generate the RSA configurations efficiently and to estimate the percolation point. In this algorithm we first create a list of pairs of neighboring vertices associated with each edge on the lattice. For each realization of the RSA process, we generate a random ordering of this list. In the usual NZ algorithm we go down this list one pair at a time and add the bonds to the system, using the "find" routine to find the roots of the two clusters at the ends of the new bond and then the "union" step to join two clusters if their roots are currently marked as distinct. Ordering the bonds beforehand is very useful for the RSA problem, since we can just go down this list, thus considering each bond just once. For the restricted-valence models, we modify this program to keep track of the valence of each site and only occupy a bond if the valences of its two sites are less than $V_{\max }$.

We study lattices of linear size $L=32,48,64,96,128$, $192,256,384,512$, and 768 corresponding to $N=(q / 2) L^{2}$ bonds, where $q=4$ (6) for square (triangular) lattices. To estimate the properties of interest we average over $10^{6}$ independent realizations starting from an empty lattice.

\section{RESULTS}

In this section we report results for the percolation threshold, critical exponents, and jamming coverage for restricted-valence RSA on the square and triangle lattices. The properties of interest are exhibited as functions of the control parameter $p$, i.e., the fraction of bonds visited. In percolation, the order parameter is usually defined as [43]

$$
\Omega(p, L) \equiv\left\langle s_{\max }\right\rangle / L^{d},
$$

where $s_{\max }$ is the number of sites in the largest cluster, $d$ is the dimensionality, and the angular brackets denote an average over realizations. The percolation threshold of the infinite lattice $p_{c}^{\infty}$ is estimated via finite-size scaling (FSS) analysis [1] and is expected to follow

$$
p_{c}^{\infty}-p_{c}(L) \sim L^{-1 / v},
$$

where $p_{c}(L)$ is the pseudocritical value for lattice size $L$ and $v$ is the critical exponent governing the correlation length. The pseudocritical value is commonly determined through the position of the maximum of some "diverging" quantity, or of a crossing point for different system sizes. In this work we analyze a set of seven quantities; five are moment ratios of the form

$$
Q_{i r}^{j s}(p, L) \equiv \frac{M_{n}(p, L)}{M_{r}^{i}(p, L) M_{s}^{j}(p, L)} \quad \text { with } \quad i r+j s=n,
$$

where the moments $M_{k}$ are defined as $M_{k}(p, L) \equiv\left\langle s^{k}\right\rangle$ and $k=1, \ldots, 4$,

$$
\begin{array}{ll}
Q_{11}^{11}=\frac{M_{2}}{M_{1}^{2}}, & Q_{12}^{\frac{1}{2} 2}=\frac{M_{3}}{M_{2}^{3 / 2}}, \quad Q_{12}^{12}=\frac{M_{4}}{M_{2}^{2}}, \\
Q_{21}^{11}=\frac{M_{3}}{M_{1}^{3}}, & Q_{12}^{11}=\frac{M_{3}}{M_{1} M_{2}} .
\end{array}
$$

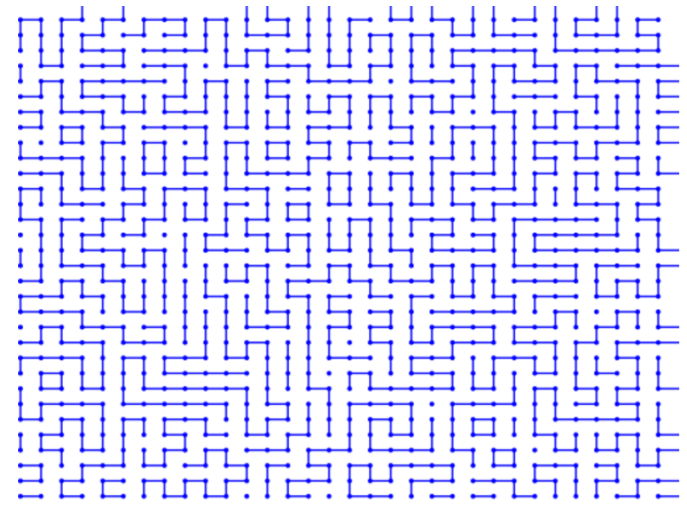

(a)

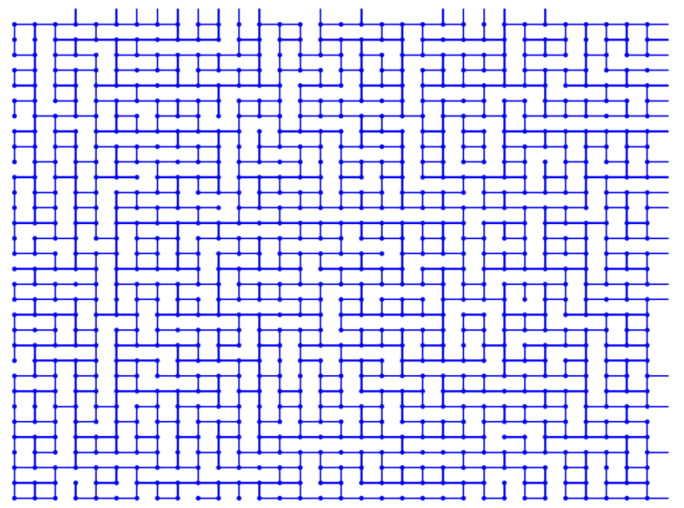

(b)

FIG. 2. Typical jammed configurations on a $32 \times 32$ lattice for $V_{\max }=2(\mathrm{a})$ and $V_{\max }=3$ (b). 
We also study the second cumulant $K_{2}(p, L)$ :

$$
K_{2}(p, L)=L^{-2}\left(M_{2}-M_{1}^{2}\right) .
$$

This property, also called the susceptibility $\chi(p, L)$, provides information about the fluctuations in the average size of all the clusters. Finally, we analyze $M_{2}^{\prime}(p, L)$, defined as the difference between the second moment of $s$, denoted by $M_{2}(p, L)$, and the mean fraction of sites in the largest cluster:

$$
M_{2}^{\prime}(p, L)=\frac{1}{L^{d}}\left\langle\sum_{i \mid s_{i}<s_{\max }} s_{i}^{2}\right\rangle=M_{2}(p, L)-\frac{\left\langle s_{\max }^{2}\right\rangle}{L^{2}},
$$

where

$$
M_{2}(p, L)=\sum_{s} s^{2} n_{s}=\frac{1}{L^{2}} \sum_{i} s_{i}^{2} .
$$

In Eqs. (5) and (6), $s_{i}$ denotes the size of cluster $i$ and $n_{s}$ the average number of clusters of size $s$.

The FSS theory of percolating systems [44] states that at the critical point $p=p_{c}^{\infty}, \Omega(p, L), M_{2}^{\prime}(p, L)$, and $K_{2}(p, L)$ obey the relations

$$
\begin{aligned}
\Omega\left(p_{c}^{\infty}, L\right) & \sim L^{-\beta / v} \mathcal{U}\left(\varepsilon L^{1 / v}\right), \\
M_{2}^{\prime}\left(p_{c}^{\infty}, L\right) & \sim L^{-\gamma / v} \mathcal{M}\left(\varepsilon L^{1 / v}\right),
\end{aligned}
$$

and

$$
K_{2}\left(p_{c}^{\infty}, L\right) \sim L^{-\gamma / v} \mathcal{K}\left(\varepsilon L^{1 / v}\right),
$$

where $\beta, \gamma$, and $v$ are critical exponents, and $\varepsilon \equiv p-p_{c}$ represents the distance to the critical point. The scaling functions $\mathcal{U}(x), \mathcal{M}(x)$, and $\mathcal{K}(x)$ exhibit universal behavior.

\section{A. Square lattice}

\section{Determination of $p_{c}^{\infty}$}

As mentioned in Sec. II, for maximum valence $V_{\max }=1$ on the square lattice there is no percolation. For $V_{\max }=2 \mathrm{a}$ typical jammed configuration is shown in Fig. 2(a). In this case there is no percolation for large systems, as shown in the Appendix. On the other hand, for $V_{\max }=3$ [see Fig. 2(b)], there are large connected regions.

Results for the properties mentioned above (for $V_{\max }=3$ ) are shown in Fig. 3. The abrupt increase of $\Omega(p, L)$ [Fig. 3(a)] suggests a phase transition at some value of $p$ between 0.40 and 0.60. Figure 3(b) shows an expanded plot of the order parameter multiplied by the factor $L^{\beta / v}$, where $\beta=5 / 36$ and $v=4 / 3$ correspond to the standard two-dimensional percolation critical exponents. A crossing point is evident at $p \approx 0.532$. Figure 3(c), which shows the moment ratio $Q_{12}^{12}$, again suggests a continuous transition with a crossing point for $p \approx 0.53$. The panel (d) shows that $Q_{11}^{11}$ exhibits similar behavior. The moment ratios exhibit strong finite-size effects, with the pseudocritical values of $Q$ increasing substantially with system size. Figure 3(e) shows the second cumulant $K_{2}$, which also exhibits signatures of a continuous transition. The peaks occur in the range $0.51<p<0.54$, in agreement with the other properties. Panel (f) shows the second cumulant scaled by $L^{\gamma / \nu}$, with $\gamma=43 / 18$ the critical exponent that governs the fluctuations in the largest cluster size. Although we observe some discrepancies for $L=32$ and 48, the curves for
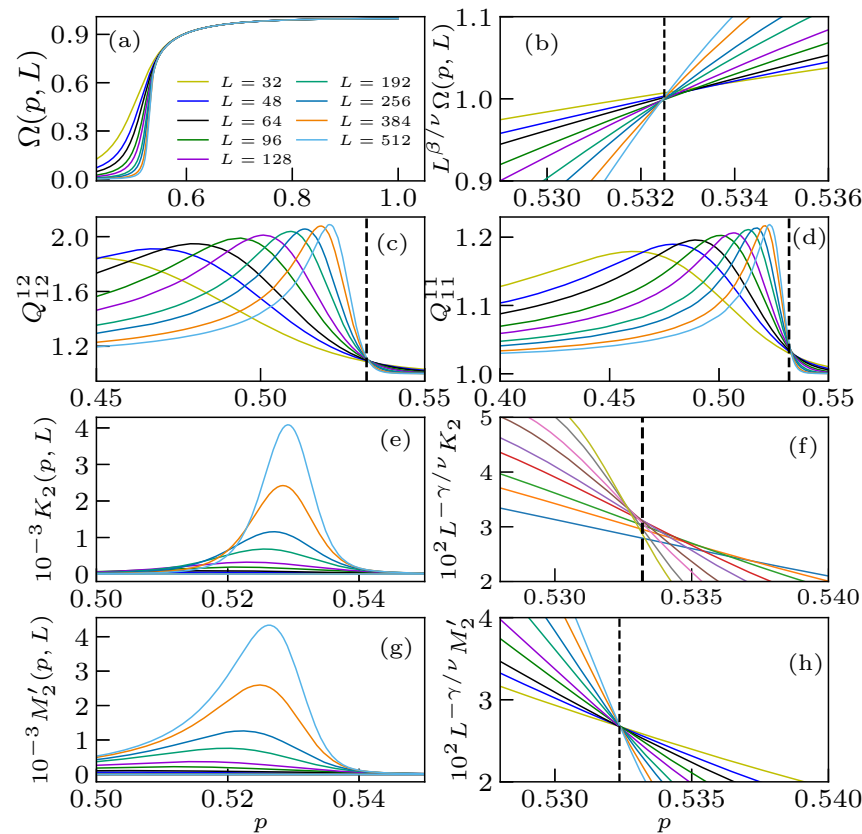

FIG. 3. Results for $V_{\max }=3$. (a) Order parameter $\Omega(p, L)$ vs $p$, with system size increasing from left to right. (b) $L^{\beta / v} \Omega$ vs $p$ near the crossing point (vertical line); (c), (d) moment ratios $Q_{12}^{12}$ and $Q_{11}^{11}$, respectively, vs $p$. The system size decreases from right to left. (e) $K_{2}$ as function of $p$ and (f) detail of the crossing region of the scaled $K_{2}$; the dashed line marks the crossing point; $(\mathrm{g})$, (h) similar to (f) and (e), but for $M_{2}^{\prime}$. In (e) and (g), system size increases from bottom to top.

larger systems intersect at $p \approx 0.532 . M_{2}^{\prime}$, plotted in Fig. $3(\mathrm{~g})$, follows the same tendencies as the other properties, exhibiting maxima that increase systematically with system size. The

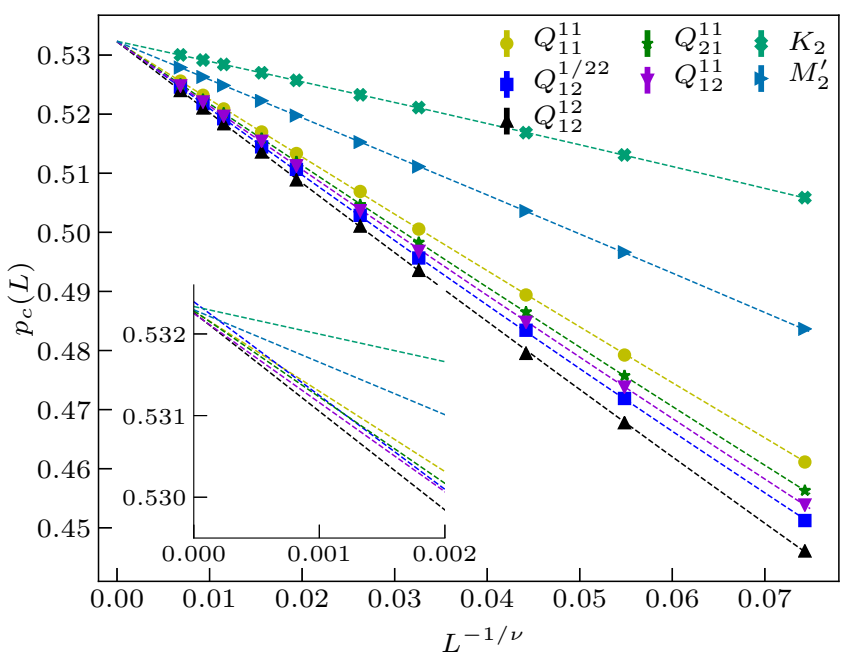

FIG. 4. Square lattice: FSS of pseudocritical points. Symbols represent simulation values and lines linear fits. Error bars are smaller than symbols. Different symbols correspond to pseudocritical points obtained from analysis of (bottom to top) $Q_{12}^{12}, Q_{12}^{1 / 22}, Q_{12}^{11}$, $Q_{21}^{11}, Q_{11}^{11}, M_{2}^{\prime}$, and $K_{2}$. Inset: Detail of the limiting $(L \rightarrow \infty)$ region. 
TABLE I. For $V_{\max }=3$, estimates for the pseudocritical points for each system size $L$ and quantity analyzed. The final line reports extrapolated $(L \rightarrow \infty)$ estimates for the critical point. Numbers in parentheses denote uncertainties.

\begin{tabular}{|c|c|c|c|c|c|c|c|}
\hline$L$ & $Q_{11}^{11}$ & $Q_{12}^{12}$ & $Q_{12}^{\frac{1}{2} 2}$ & $Q_{21}^{11}$ & $Q_{12}^{11}$ & $K_{2}$ & $M_{2}^{\prime}$ \\
\hline 32 & $0.46112(1)$ & $0.4460(1)$ & $0.45122(2)$ & $0.45629(5)$ & $0.45385(3)$ & $0.5058(1)$ & $0.48366(4)$ \\
\hline 48 & $0.47923(1)$ & $0.4677(1)$ & $0.47188(4)$ & $0.4757(1)$ & $0.47381(6)$ & $0.51310(4)$ & $0.49663(4)$ \\
\hline 64 & $0.489401(9)$ & $0.4795(1)$ & $0.48343(2)$ & $0.48650(6)$ & $0.48477(4)$ & $0.51687(4)$ & $0.50362(2)$ \\
\hline 96 & $0.500547(6)$ & $0.4935(1)$ & $0.49565(1)$ & $0.49828(4)$ & $0.49678(4)$ & $0.52112(4)$ & $0.51112(1)$ \\
\hline 128 & $0.506917(6)$ & $0.50105(8)$ & $0.50287(1)$ & $0.50466(4)$ & $0.50366(3)$ & $0.52327(2)$ & $0.51526(1)$ \\
\hline 192 & $0.513341(6)$ & $0.50887(8)$ & $0.51066(1)$ & $0.51192(3)$ & $0.51126(2)$ & $0.52572(2)$ & $0.51976(1)$ \\
\hline 256 & $0.516989(8)$ & $0.51359(5)$ & $0.51446(1)$ & $0.51588(3)$ & $0.51541(1)$ & $0.52700(3)$ & $0.522269(9)$ \\
\hline 384 & $0.520888(3)$ & $0.51837(4)$ & $0.519221(5)$ & $0.52015(2)$ & $0.51964(1)$ & $0.52842(1)$ & $0.524864(8)$ \\
\hline 512 & $0.523199(3)$ & $0.52104(5)$ & $0.52177(1)$ & $0.52248(2)$ & $0.52207(1)$ & $0.52916(1)$ & $0.526303(8)$ \\
\hline 768 & $0.525601(4)$ & $0.52400(3)$ & $0.524578(7)$ & $0.525048(1)$ & $0.52478(1)$ & $0.53003(1)$ & $0.527896(6)$ \\
\hline$\infty$ & $0.5322(1)$ & $0.5323(1)$ & $0.5322(1)$ & $0.53228(4)$ & $0.5322(1)$ & $0.53233(3)$ & $0.53222(5)$ \\
\hline
\end{tabular}

peaks appear in the range $0.50<p<0.54$. The scaling plot (h) shows a crossing at $p=0.532$.

FSS analysis of the pseudocritical points, shown in Fig. 4, reveals that for each property, $p_{c}(L)$ is well fit by a straight line when plotted versus $L^{-1 / v}$. Here the pseudocritical points $p_{c}$ for each quantity are estimated using a polynomial fit to approximately 10 points around the global maximum. (The order of the polynomial is chosen as the lowest order that yields residuals without systematic behavior. In most cases fourth-order polynomials are used.) The uncertainty in the position of the maximum was estimated through the rootmean-square deviation (RMSD); in the worst case, the RMSD $\sim 10^{-4}$ (see Table I).

In the limit $L \rightarrow \infty$ all the estimates for the percolation threshold converge to very similar values (see Fig. 4 and
Table I). The final estimate for the percolation threshold $p_{c}^{\infty}$ is obtained through a weighted average of the estimates associated with each property with weights $\propto 1 / \sigma^{2}$, where $\sigma$ represents the uncertainty of each estimate, yielding a final estimate of $p_{c}^{\infty}=0.5323(1)$ for $V_{\max }=3$ on the square lattice.

The results for the scaled values of $\Omega, K_{2}$, and $M_{2}^{\prime}$ suggest that the transition belongs to the standard percolation universality class (SPUC). We verify this conclusion via datacollapse analyses, as shown in Fig. 5. Panel (a) shows that the SPUC critical exponents yield a good collapse of the simulation data for different system sizes. The log-log plot of the order parameter [inset (a)] provides $\beta / v=0.109(3)$, quite close to the exact value, $\beta / v=0.104166 \ldots$ [1], for SPUC in two dimensions. The data collapse of $M_{2}^{\prime}$ [see panel
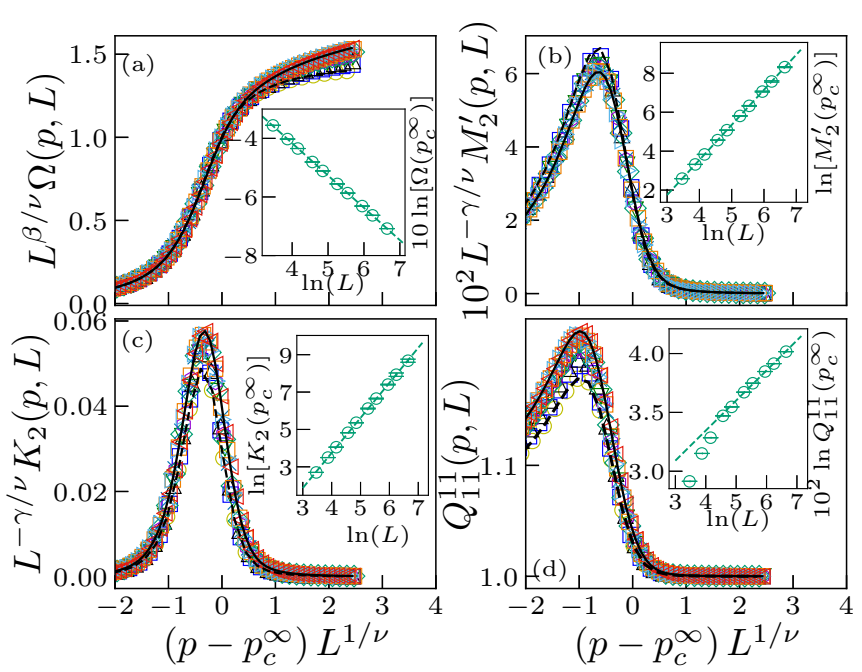

FIG. 5. Panels (a), (b), (c), and (d) exhibit data collapses for $\Omega, M_{2}^{\prime}, K_{2}$, and $Q_{11}^{11}$, respectively. Insets are log-log plots of the aforementioned properties at the critical point. Exact critical exponent values for standard percolation in two dimensions are used in the data-collapse analysis. Symbols $\circ, \square, \triangle, \nabla, \square, \diamond, \times, \square, \triangleright$, $\triangleleft$ correspond to system sizes $L=32,48,64,96,128,192,256$, 384,512 , and 768, respectively. Dashed (solid) curves correspond to $L=32$ (768).
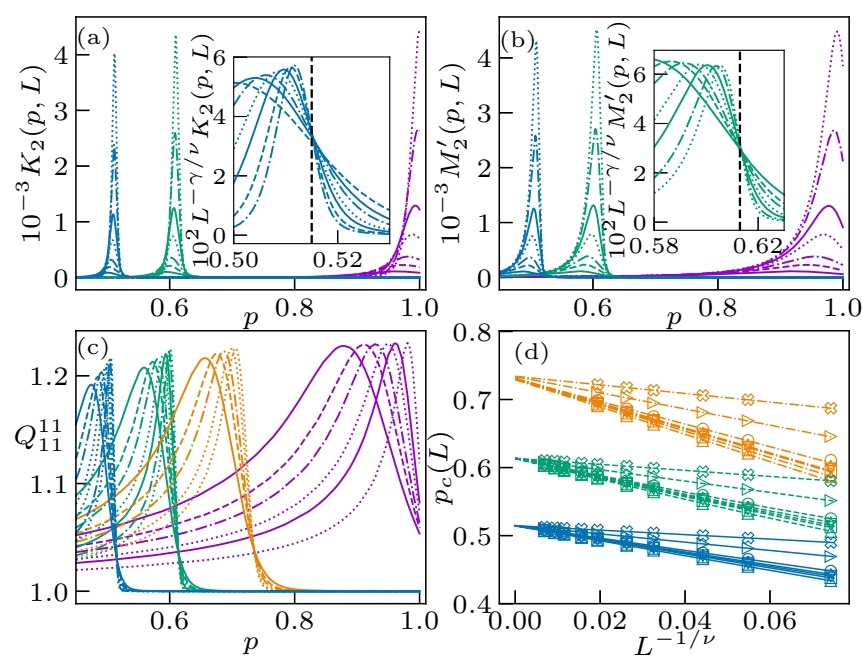

FIG. 6. Valence disorder on square lattice. (a) $K_{2}(p, L)$ vs concentration $p$. Different line patterns denote different system sizes, while the three groups of lines correspond (from left to right) to $\left(V_{\mathrm{i}}, V_{\mathrm{j}}\right)=(3,4),(2,4)$, and $(1,4)$. Case $(2,3)$ (not shown) exhibits similar behavior. The inset shows the crossing points of $K_{2}$, scaled using standard percolation exponents, for case $(3,4)$. (b) Similar to (a), but for $M_{2}^{\prime}(p, L)$ the inset is for case $(2,4)$. (c) $Q_{11}^{11}$ vs $p$ for cases (left to right) $(3,4),(2,4),(2,3)$, and $(1,4)$. (d) Pseudocritical points vs $L^{-1 / v}$ for cases $(2,3),(2,4)$ and $(3,4)$ (upper to lower). 


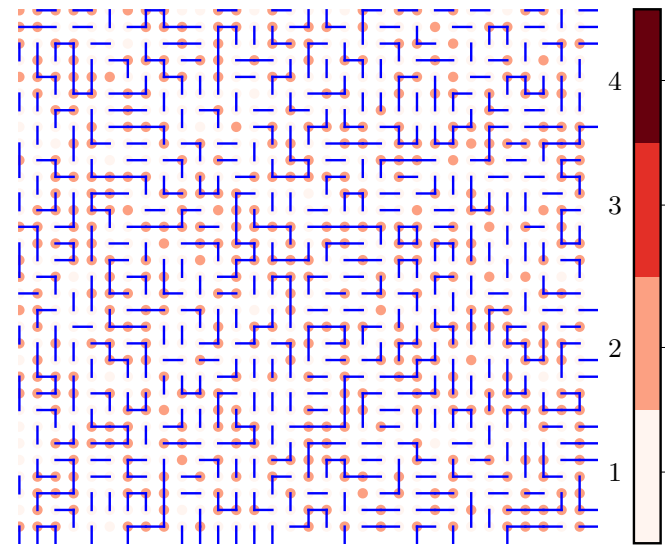

(a)

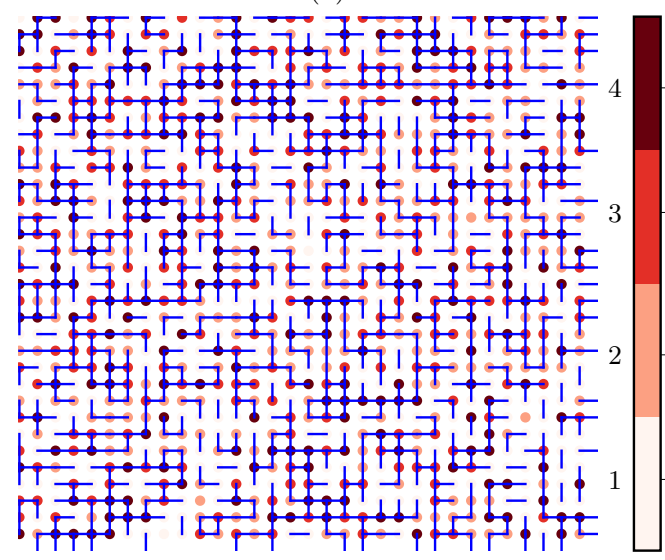

(c)

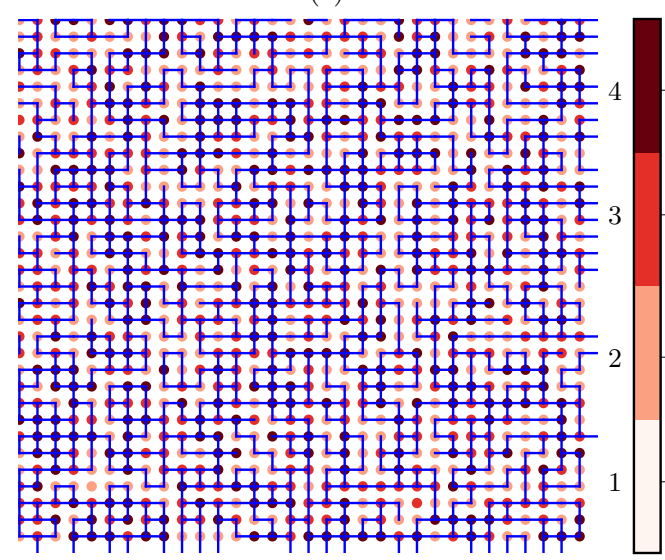

(e)

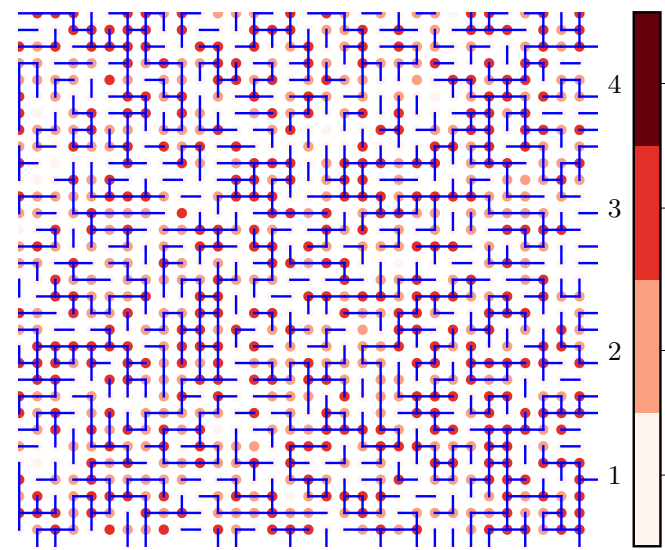

(b)

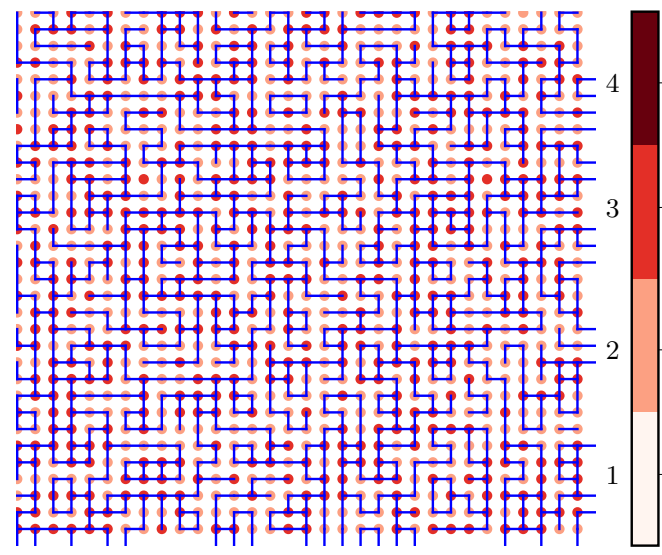

(d)

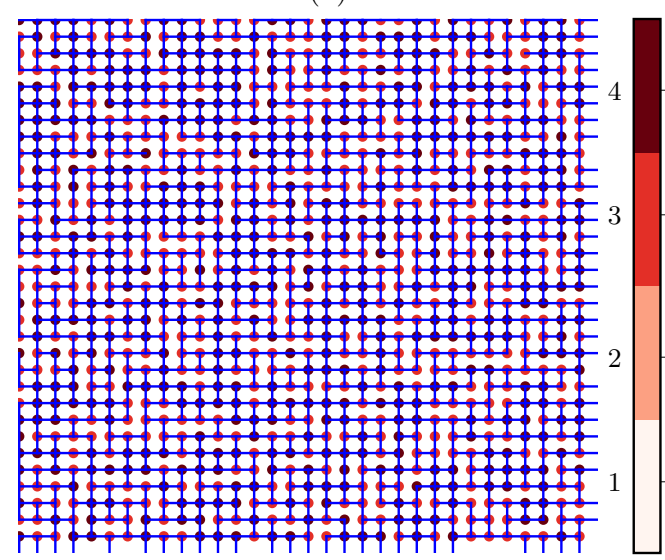

(f)

FIG. 7. Typical jammed configurations for disordered cases $(1,2),(1,3),(1,4),(2,3),(2,4)$, and $(3,4)$ (panels a-f, respectively) for $L=32$. Color scales denote valences. Solid lines denote occupied bonds.

(b)] also exhibits a good overlap, although slight deviations are observed for $L=32$ and $L=48$ in the vicinity of the critical point. The log-log plots provide a slope of $1.800(1)$, in agreement with the theoretical prediction of the ratio $\gamma / v$ for SPUC. For $K_{2}$ [see Fig. 5(c)], the data points and the log-log plots (see inset) provide a slope of 1.782(8), again in agreement with the exact result $\gamma / v=1.7916 \ldots[1]$. The ratios $\beta / \nu$ and $\gamma / \nu$ satisfy the hyperscaling relation $d \nu=\gamma+2 \beta$ within uncertainty.

\section{Valence disorder}

We now analyze restricted-valence RSA with valence disorder, in which the valences associated with each site are taken as independent, identically distributed random variables. We consider valence distributions uniform on the set $\left\{V_{\mathrm{i}}, \ldots, V_{\mathrm{j}}\right\}$. At each realization, a new set of valences is generated. On the square lattice we considered $\left(V_{\mathrm{i}}, V_{\mathrm{j}}\right)=$ $(1,4),(1,3),(1,2),(2,4),(2,3)$, and $(3,4)$, with each valence $V_{\mathrm{i}}$ occurring with equal probability. 
There is no percolation for cases $(1,3)$ and $(1,4)$. While disorder alters the percolation thresholds and jamming coverages, it does not appear to affect the critical exponents. According to the Harris criterion [45,46], disorder is relevant if $d v \leqslant 2$. Since the transition in the pure system belongs to the SPUC with $d v=2(4 / 3)>2$, disorder is indeed expected to be irrelevant. The quantities $K_{2}(p, L), M_{2}^{\prime}(p, L)$, and $Q_{11}^{11}$ in the presence of disorder are shown in Figs. 6(a), 6(b) and 6(c), respectively.

Figure 6 shows clear evidence of a continuous percolation transition, as in the pure case. The insets of Figs. 6(a) and 6(b) exhibit crossings using the standard percolation critical exponents, suggesting that disorder does not affect the universality class of the transition. Note as well that the crossing value of $Q_{11}^{11}$ is the same as for the pure case. As expected, the percolation threshold moves to lower values of $p$ as the mean valence increases. For example, for $(3,4)$ [blue lines in Figs. 6(a)-6(c)], half the sites have maximum valence 3 and half maximum valence 4 . Both valences percolate, and the percolation threshold occurs at a value between those for the pure cases.

Our estimates for the percolation threshold are obtained via FSS as shown in Fig. 6(d), providing $p_{c}^{\infty}=0.7283(5)$, $0.6099(1), 0.5120$ (2) for the cases $(2,3),(2,4)$, and $(3,4)$, respectively. The analyses follow the same lines as the pure cases $(3,3)$ and $(4,4)$. It is worth noting that although $(1,4)$ and $(2,3)$ possess the same mean valence, the latter percolates while the former does not.

\section{Coverage density and jamming state}

Without disorder, case $(1,1)$ is the most restrictive and hence the lowest jamming coverage is obtained. In this case each absorbed bond prevents all its neighbor bonds from being occupied, so that in a perfectly regular pattern corresponding to full coverage of sites, only $1 / 4$ of the bonds are occupied. Analysis of this case goes back to the work of Nord and Evans [47], who obtained a saturation coverage of 0.9068 . Subsequently, de Oliveira et al. [48] obtained 0.90677(6) via series expansions and simulation. Our approach provides $0.906814(5)$ (a deviation of less than $0.0045 \%$ compared with [48]). For $(3,3)$ the insertion of a bond blocks two of its six neighbors and thus, in a regular pattern, $3 / 4$ of bonds can be occupied. In this case we find a coverage of 0.999391(1). Case $(4,4)$ is trivial since all edges are occupied by bonds.

Typical jammed configurations for disordered cases are shown in Fig. 7. For $(1,2)$ [Fig. 7(a)], only sites with valence 1 and 2 are permitted. Since half the sites have valence 1 , there are isolated bonds and terminal points. Figure 7(c) shows $(1,4)$, which is the most heterogeneous. All possibilities are observed in the snapshot, from isolated bonds to sites with the maximum number of bonds. Case $(3,4)$ [Fig. 7(f)] has the highest density of bonds; nevertheless, there are sites with valence 4 that have only two incident bonds.

Results for the coverage density $\rho$ as a function of $p$ and the jamming coverage are shown in Fig. 8. Figure 8(a) exhibits the density of occupied bonds as a function of $p$. The red dashed line represents the limiting case $(4,4)$ in which all bonds are occupied. While the curves are generally similar, those for $(1,4)$ and $(2,2)$ cross near $p=0.60$, suggesting that the rate at
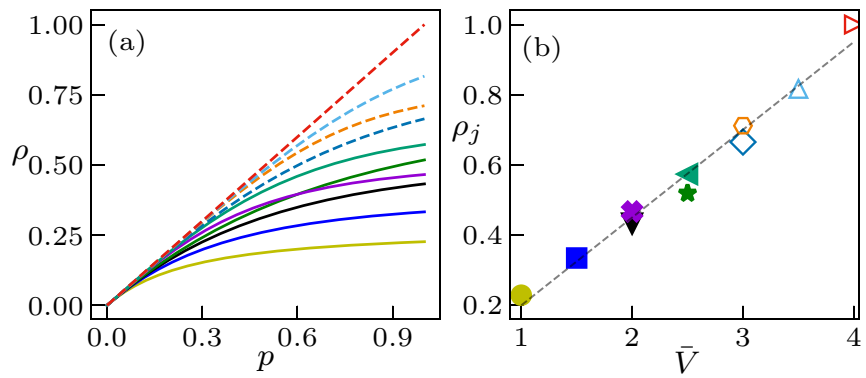

FIG. 8. (a) Coverage density $\rho$ vs $p$ for $L=128$ and distinct combinations of valences. From bottom to top, $(1,1),(1,2),(1,3)$, $(1,4),(2,2),(2,3)$ (solid lines) and $(2,4),(3,3),(3,4)$, and $(4,4)$ (dashed lines). (b) Scatter plot of the jamming fraction vs average valence. Full (open) symbols correspond to solid (dashed) lines in (a). The dashed line is a least-squares linear fit to the points.

which bonds are occupied changes in a nontrivial manner as $p$ varies. In Fig. 8(d) we plot the jamming density versus $\bar{V}$, the arithmetic average of the valencies in the range $\left\{V_{i}, \ldots, V_{j}\right\}$; $p_{j}$ increases approximately linearly with $\bar{V}$.

\section{Percolation thresholds}

We determine the bond occupation fractions at the pseudocritical percolation points $\rho_{c}(L)$, which, when extrapolated to infinite system size, furnish estimates for the bond percolation densities $\rho_{c}$ for the different cases of pure and mixed valences (see Table II). Pure valence 4 is simply bond percolation on the square lattice, and our result is consistent with the exact value, $\rho_{c}=1 / 2$ [4]. At the other extreme $(2,3), \rho_{c}$ is clearly larger than one-half. Recalling that the pure valence- 2 system does not percolate, $\rho_{c}>1 / 2$ can be understood qualitatively by noting that many occupied bonds falling in regions rich in valence- 2 sites cannot contribute to percolation. A similar observation applies to the $(2,4)$ mixture. For $(3,3)$ the deviation from $\rho_{c}=1 / 2$ is barely significant, while for $(3$, 4) our result is consistent with a percolation density of $1 / 2$. It is nonetheless surprising that the percolation density for $(3,3)$ is smaller than $1 / 2$. While we defer a detailed study of this case to future work, we note that in all cases except $(4,4)$, the occupation of bonds incident upon a given site

TABLE II. Simulation results for $p_{c}^{\infty}, p_{j}, \theta_{\text {sites }}$, and $\rho_{c}^{\infty}$ for the square lattice. The horizontal lines in the first column correspond to cases without percolation.

\begin{tabular}{lcccc}
\hline \hline$\left(V_{i}, V_{j}\right)$ & $p_{c}^{\infty}$ & $\rho_{j}$ & $\theta_{\text {sites }}$ & $\rho_{c}^{\infty}$ \\
\hline$(1,1)$ & - & $0.226705(3)$ & $0.906814(5)$ & - \\
$(1,2)$ & - & $0.332928(2)$ & $0.958746(1)$ & - \\
$(1,3)$ & - & $0.432512(6)$ & $0.982236(1)$ & - \\
$(1,4)$ & - & $0.517817(6)$ & $0.992235(2)$ & - \\
$(2,2)$ & - & $0.465780(5)$ & $0.987851(9)$ & - \\
$(2,3)$ & $0.7283(5)$ & $0.572630(2)$ & $0.995678(9)$ & $0.512(2)$ \\
$(2,4)$ & $0.6099(1)$ & $0.664237(1)$ & $0.998557(9)$ & $0.5070(6)$ \\
$(3,3)$ & $0.5323(1)$ & $0.710935(1)$ & $0.999391(1)$ & $0.496(2)$ \\
$(3,4)$ & $0.5120(2)$ & $0.816074(5)$ & $0.999903(3)$ & $0.498(3)$ \\
$(4,4)$ & $0.5000(1)$ & 1.00000000 & 1.00000000 & $0.5000(1)$ \\
\hline \hline
\end{tabular}


TABLE III. Estimates for the pseudocritical points and other quantities for each system size $L$ for $(4,4)$. The final line reports extrapolated $(L \rightarrow \infty)$ estimates for critical values. Numbers in parentheses denote uncertainties.

\begin{tabular}{|c|c|c|c|c|c|c|c|}
\hline$L$ & $Q_{11}^{11}$ & $Q_{12}^{12}$ & $Q_{12}^{\frac{1}{2} 2}$ & $Q_{21}^{11}$ & $Q_{12}^{11}$ & $K_{2}$ & $M_{2}^{\prime}$ \\
\hline 32 & $0.30009(3)$ & $0.2887(1)$ & $0.29211(4)$ & $0.297392(4)$ & $0.29445(6)$ & $0.33240(2)$ & $0.319581(2)$ \\
\hline 48 & $0.31363(2)$ & $0.3050(2)$ & $0.30789(4)$ & $0.307626(2)$ & $0.30875(7)$ & $0.33753(9)$ & $0.328151(1)$ \\
\hline 64 & $0.32089(2)$ & $0.3148(1)$ & $0.31601(2)$ & $0.318307(1)$ & $0.31754(7)$ & $0.34056(3)$ & $0.3311319(5)$ \\
\hline 96 & $0.32892(2)$ & $0.3234(2)$ & $0.32586(3)$ & $0.327910(1)$ & $0.32617(5)$ & $0.34345(6)$ & $0.3367670(4)$ \\
\hline 128 & $0.33325(1)$ & $0.3292(1)$ & $0.3301(4)$ & $0.332074(1)$ & $0.33126(4)$ & $0.34520(1)$ & $0.3393849(7)$ \\
\hline 192 & $0.33817(1)$ & $0.3350(1)$ & $0.3361(4)$ & $0.336908(1)$ & $0.33636(3)$ & $0.34706(1)$ & $0.3425698(7)$ \\
\hline 256 & $0.34082(1)$ & $0.33839(9)$ & $0.3393(3)$ & $0.340100(1)$ & $0.33937(4)$ & $0.34795(1)$ & $0.3445013(4)$ \\
\hline 384 & $0.34364(1)$ & $0.34190(8)$ & $0.3424(1)$ & $0.3429443(9)$ & $0.34273(2)$ & $0.34903(9)$ & $0.3464356(3)$ \\
\hline 512 & $0.34528(1)$ & $0.34375(7)$ & $0.34433(1)$ & $0.3447238(7)$ & $0.34447(1)$ & $0.34964(2)$ & $0.3475288(7)$ \\
\hline 768 & $0.34705(1)$ & $0.34584(4)$ & $0.3462(1)$ & $0.346731(3)$ & $0.346494(1)$ & $0.35030(7)$ & $0.348957(9)$ \\
\hline$\infty$ & $0.3518(5)$ & $0.3516(3)$ & $0.3518(5)$ & $0.35172(1)$ & $0.35177(7)$ & $0.3519(7)$ & $0.35175(1)$ \\
\hline
\end{tabular}

are not independent events, so that deviations from standard independent percolation are possible in principle.

We summarize our results for the percolation threshold, jamming coverage, and the density of occupied sites at jamming for the square lattice in Table II.

\section{B. Triangle lattice}

We adopt the same approach as employed for the square lattice; the scaling behaviors are quite similar, hence we only report numerical values. For $(1,1),(2,2),(1,2)$, and $(1,3)$, valence restrictions prohibit percolation. All other cases exhibit a percolation transition characterized by size-dependent peaks in $M_{2}^{\prime}, K_{2}$, and the $Q_{i r}^{j s}$. Table III exhibits simulation values for case $(4,4)$.

TABLE IV. Simulation results for $p_{c}^{\infty}, p_{j}, \theta_{\text {sites }}$, and $\rho_{c}^{\infty}$ for the triangle lattice. The horizontal lines in the first column correspond to cases without percolation.

\begin{tabular}{lcccc}
\hline \hline$\left(V_{i}, V_{j}\right)$ & $p_{c}^{\infty}$ & $\rho_{j}$ & $\theta_{\text {sites }}$ & $\rho_{c}^{\infty}$ \\
\hline$(1,1)$ & - & $0.152338(1)$ & $0.914028(2)$ & \\
$(1,2)$ & - & $0.229197(3)$ & $0.966224(2)$ & \\
$(1,3)$ & - & $0.302104(5)$ & $0.987364(7)$ & \\
$(1,4)$ & $0.8582(5)$ & $0.371539(2)$ & $0.995508(5)$ & $0.35(3)$ \\
$(1,5)$ & $0.6374(5)$ & $0.434392(1)$ & $0.998354(5)$ & $0.35(1)$ \\
$(1,6)$ & $0.5489(4)$ & $0.488033(5)$ & $0.999309(9)$ & $0.361(3)$ \\
$(2,2)$ & - & $0.312030(7)$ & $0.983257(6)$ & \\
$(2,3)$ & $0.6133(8)$ & $0.388466(6)$ & $0.993781(7)$ & $0.347(1)$ \\
$(2,4)$ & $0.4651(6)$ & $0.460755(9)$ & $0.997971(2)$ & $0.346(2)$ \\
$(2,5)$ & $0.4217(8)$ & $0.526968(8)$ & $0.999383(2)$ & $0.348(3)$ \\
$(2,6)$ & $0.4017(4)$ & $0.583207(5)$ & $0.999797(8)$ & $0.346(2)$ \\
$(3,3)$ & $0.3980(1)$ & $0.472670(3)$ & $0.997277(9)$ & $0.338(2)$ \\
$(3,4)$ & $0.3715(9)$ & $0.548710(9)$ & $0.999181(1)$ & $0.341(1)$ \\
$(3,5)$ & $0.3625(0)$ & $0.619447(1)$ & $0.999802(7)$ & $0.343(1)$ \\
$(3,6)$ & $0.3582(8)$ & $0.678423(6)$ & $0.999952(4)$ & $0.344(1)$ \\
$(4,4)$ & $0.3517(1)$ & $0.635157(1)$ & $0.999720(1)$ & $0.3438(5)$ \\
$(4,5)$ & $0.3496(5)$ & $0.710952(4)$ & $0.999948(9)$ & $0.3455(3)$ \\
$(4,6)$ & $0.3488(4)$ & $0.776258(6)$ & $0.999993(5)$ & $0.3464(9)$ \\
$(5,5)$ & $0.3475(1)$ & $0.802231(3)$ & $0.999990(1)$ & $0.346(2)$ \\
$(5,6)$ & $0.3474(7)$ & $0.876628(1)$ & $0.999999(1)$ & $0.347(1)$ \\
$(6,6)$ & $0.3472(5)$ & 1.00000000 & 1.00000000 & $0.3472(5)$ \\
\hline \hline
\end{tabular}

In the limiting case $(6,6)$, the percolation threshold is known exactly: $p_{c}=2 \sin (\pi / 18)=0.347296355 \ldots$ [22]. Our estimate of $p_{c}^{\infty}(6,6)=0.3472(5)$ is in agreement with this result. Estimates for the other cases are shown in Table IV. Analysis of critical exponents again yields values consistent with SPUC. For example, scaling plots of $M_{2}^{\prime}$ and $\Omega$ using SPUC exponents exhibit crossings of curves at the percolation threshold.

The jamming probability and coverage density are determined via the same techniques used for the square lattice. The curves for the density of occupied bonds for $(2,5)$ and $(2,6)$ cross the curve for $(3,3)$, similar to cases $(1,4)$ and $(2,2)$ on the square lattice. A compendium of results for the triangle lattice is reported in Table IV. Different from the square lattice, here all percolation thresholds are greater than or equal to the exact value for the unrestricted triangle lattice.

\section{CONCLUSIONS}

We investigate restricted-valence random sequential adsorption in its pure and disordered versions on the square and triangle lattices. We show that there is no percolation for $V_{\max }=2$ on the square lattice. In other cases, Monte Carlo simulations coupled with the Newman-Ziff algorithm are used, revealing a continuous transition for cases $(2,3),(2,4)$, $(3,3),(3,4)$, and $(4,4)$. Finite-size scaling analysis of $M_{2}^{\prime}, K_{2}$, and $Q_{i r}^{j s}$ is employed to estimate the percolation threshold. Scaling analyses show that the critical exponents are in good agreement with the standard percolation universality class, as one might expect given that the correlations are local. In the disordered cases, the universality class is preserved although the percolation threshold naturally depends on the average valence.

The coverage densities and jamming coverages are estimated for all cases. On the square lattice, our result for the jamming of dimer RSA, $\theta=0.906814(5)$, is in agreement with previous estimates of Nord and Evans [47] and of de Oliveira and co-workers [48], and surpasses their precision. The jamming fraction was estimated for all cases, with uncertainties on the order of $10^{-6}$. Our estimates for percolation thresholds in restricted-valence cases (i.e., some fraction of sites having $\left.V_{\max }<4\right)$ are $\geqslant 1 / 2$, as expected, except for $(3,3)$, 
where we find $p_{c}=0.496(2)$. For the triangle lattice, our estimate for $p_{c}$ is again in agreement with the known exact value. The largest estimate for $p_{c}$ is for $(1,4)$, with $p_{c}^{\infty}=0.8582(5)$. Our study also provides an estimate for the jamming coverage of dimer RSA on the triangle lattice: $\theta=0.914028(2)$. This is consistent with, and considerable more precise than, the previous estimate of 0.9142(12) obtained by Perino et al. [49]. Our results for the triangle lattice are again consistent with the standard percolation universality class.

We expect our results to be of use in interpreting experiments on irreversible deposition on substrates of reduced functionality, and/or with disorder, and to the question of percolation of the deposited structure. Our study of percolation in mixed cases suggests several avenues for future work. It should be possible, if perhaps challenging, to demonstrate mathematically that on the square lattice, the $(1,4)$ mixture does not percolate whereas the $(2,3)$ mixture does. More generally, identifying the percolation threshold surface on the simplex $f_{1}+f_{2}+f_{3}+f_{4}=1$ (where the $f_{i}$ denote site fractions with maximum valence $i$ in a random mixture) is an outstanding challenge. Similar open questions exist for the triangle lattice.

\section{ACKNOWLEDGMENTS}

A.F., D.C.S., and R.D. and acknowledge financial support from CNPq, CAPES, and Fapemig, Brazil. R.D. acknowledges support from $\mathrm{CNPq}$, Brazil under Project No. 303766/2016-6.

\section{APPENDIX: NO PERCOLATION FOR $V_{\max }=2$}

Consider a final configuration of dimer deposition on $\mathbb{Z}^{2}$ with $V_{\max }=2$. Let $C$ be the set of sites connected to the origin, $\mathcal{O}$. $C$ is the union of sites that are $1,2, \ldots, n$ steps from $\mathcal{O}$, along the path of occupied edges linking the sites to $\mathcal{O}$. Let $\mathrm{X}_{j} \in\{0,1,2\}$ be the number of sites exactly $j$ steps from $\mathcal{O}$ in $C$. (In case $C$ is a closed loop, we take $j$ as the minimum number of steps to $\mathcal{O}$.) We note that $\mathrm{X}_{j}$ is a nonincreasing sequence, with $\mathrm{X}_{j}=0$ for $j>n$. Let $P(n)$ be the probability, over the space of all final configurations, that the maximum number of steps from $\mathcal{O}$ in the cluster containing the origin is $n$. We aim to show that $\exists c<1$ such that $P(n)<c^{n}$, so that $\mathbb{E} n<\infty$, i.e., there is no percolation.

Let $A_{j}$ be the event $\mathrm{X}_{j}=2$. We show that $P\left(A_{j+1} \mid A_{j}\right)<1$. Given $A_{j}$, let $x_{j}$ be one of the sites that are $j$ steps from $\mathcal{O}$, let $\mathbf{z}_{1}, \mathbf{z}_{2}$, and $\mathbf{z}_{3}$ be the sites neighboring $\mathbf{x}_{j}$ distinct from $\mathbf{x}_{j-1}$, and let $y_{i}$ be the edge linking sites $\mathbf{x}_{j}$ and $\mathbf{z}_{i}$. Let $B_{j}$ be the event that edges $y_{1}, y_{2}$, and $y_{3}$ are all unoccupied in the final configuration. Occurrence of $B_{j}$ implies that $\mathrm{X}_{j+1}<2$. Thus, $P\left(B_{j}\right)>0$ implies $P\left(A_{j+1} \mid A_{j}\right)<1$.

We now argue that $P\left(B_{j}\right)>0$ for any $j>0$. A sufficient condition for occurrence of $B_{j}$ is that sites $\mathbf{z}_{1}, \mathbf{z}_{2}$, and $\mathbf{z}_{3}$ are all of valence 2 via edges $y_{m} \notin\left\{y_{1}, y_{2}, y_{3}\right\}$. An example of such a configuration is shown in Fig. 9. Recall that each edge $y$ is assigned a real time $t_{y} \in(0,1]$. Initially all edges are empty, and edge $y$ becomes occupied at time $t_{y}$ if permitted by the maximum-valence rules; otherwise it remains empty for all time.

Consider a site $\mathbf{s}$ and let $u_{1}, \ldots, u_{4}$ be the edges incident on $\mathbf{s}$, with $t_{1}, \ldots, t_{4}$ their associated times. Call $e(\mathbf{s})$ the pair of

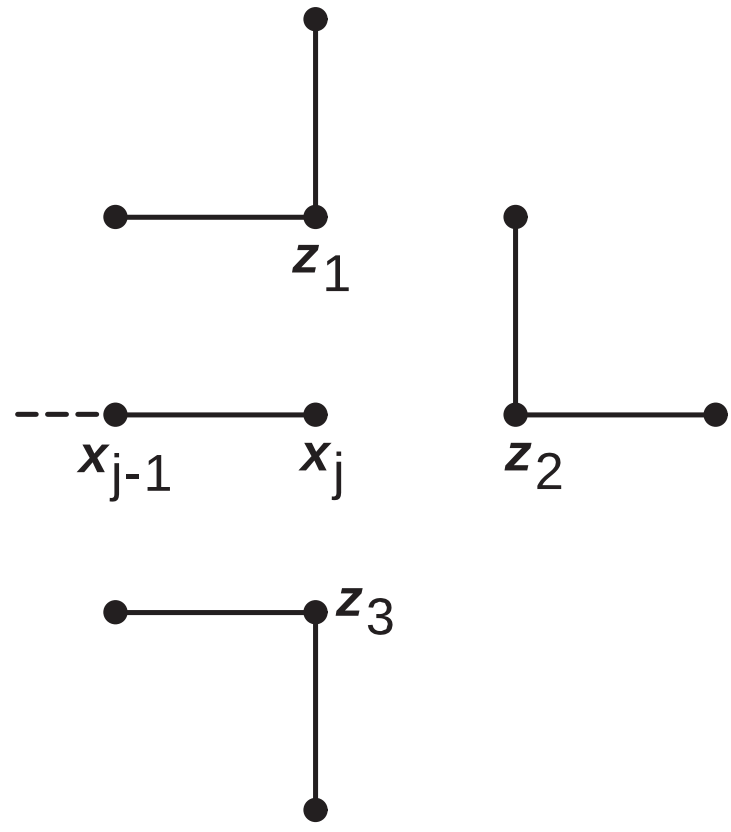

FIG. 9. A configuration corresponding to event $B_{j}$.

edges $u_{i}$ having the smallest and second-smallest times. For an arbitrary edge $y$, let $\mathbf{s}_{1}$ and $\mathbf{s}_{2}$ be its terminal sites. A sufficient (but not necessary) condition for $y$ to be occupied in the final configuration is that $y$ belong to $e\left(\mathbf{s}_{1}\right)$ and to $e\left(\mathbf{s}_{2}\right)$. Using the fact that the $t_{j}$ are i.i.d. uniformly distributed on $(0,1]$, one readily verifies that $\operatorname{Prob}\left[y \in e\left(\mathbf{s}_{1}\right) \cap e\left(\mathbf{s}_{2}\right)\right]=13 / 35$.

Now consider the local configuration shown in Fig. 10. A sufficient condition for the edges indicated by solid lines to be occupied is that they belong to the sets $e$ associated with

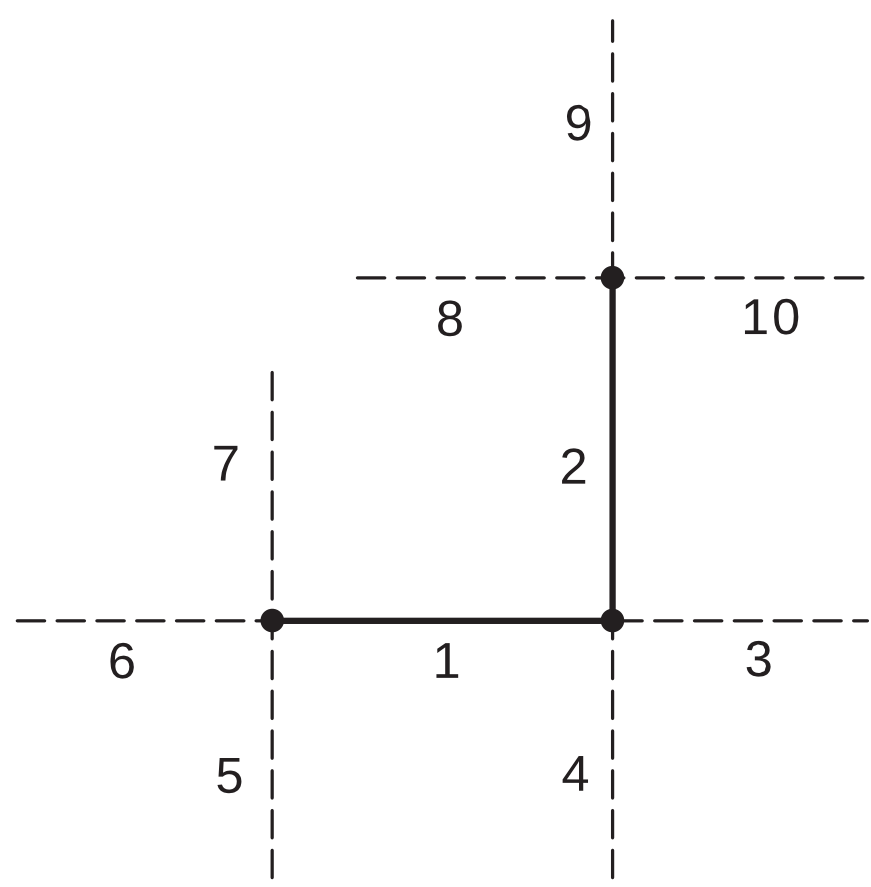

FIG. 10. Part of a configuration corresponding to event $B_{j}$. Numbers label the edges and associated times. 
the three sites upon which these edges are incident; these conditions naturally imply certain restrictions on the $t_{i}$. Integrating over the $t_{i}$, considering the diverse orderings allowed by the above-mentioned constraints we find the probability of the two-edge configuration shown in Fig. 10 to be at least $87 / 900 \equiv b^{1 / 3}$. It follows that $P\left(B_{j}\right)>b>0$. By the same argument, if $X_{j}=1$, there is a nonzero probability that $X_{j+1}=$ 0 . Thus there is a finite constant $C$ such that $\operatorname{Prob}\left[X_{n}>0\right]<$ $C(1-b)^{n}$, implying that the percolation probability is zero. Although the argument is for the square lattice, it can be adapted via straightforward modification to arbitrary lattices of finite degree.
[1] D. Stauffer and A. Aharony, Introduction to Percolation Theory, 2nd ed. (CRC Press, Boca Raton, FL, 1994).

[2] J. Feder, J. Theor. Biol. 87, 237 (1980).

[3] A. Rényi, Publ. Math. Inst. Hung. Acad. Sci. 3, 109 (1958).

[4] D. Stauffer, J. Phys. A: Math. Gen. 19, 3933 (1986).

[5] T. Odagaki and S. Toyofuku, J. Phys.: Condens. Matter 10, 6447 (1998).

[6] C. L. Henley, Phys. Rev. Lett. 71, 2741 (1993).

[7] A. Bunde, S. Havlin, and M. Porto, Phys. Rev. Lett. 74, 2714 (1995).

[8] P. J. Flory, J. Am. Chem. Soc. 63, 3083 (1941).

[9] P. J. Flory, J. Am. Chem. Soc. 63, 3091 (1941).

[10] P. J. Flory, J. Am. Chem. Soc. 63, 3096 (1941).

[11] J. Machta, Phys. Rev. Lett. 66, 169 (1991).

[12] K. Moon and S. M. Girvin, Phys. Rev. Lett. 75, 1328 (1995).

[13] T. S. Ray and N. Jan, Phys. Rev. Lett. 72, 4045 (1994).

[14] J. W. Evans, Rev. Mod. Phys. 65, 1281 (1993).

[15] S. Torquato and F. H. Stillinger, Rev. Mod. Phys. 82, 2633 (2010).

[16] G. Kondrat and A. Pękalski, Phys. Rev. E 63, 051108 (2001).

[17] B. Bonnier, M. Hontebeyrie, Y. Leroyer, C. Meyers, and E. Pommiers, Phys. Rev. E 49, 305 (1994).

[18] M. Cieśla, P. Kubala, and W. Nowak, Phys. A 527, 121361 (2019).

[19] P. Kubala, M. Cieśla, and R. M. Ziff, Phys. Rev. E 100, 052903 (2019).

[20] E. Roman and N. Majlis, Solid State Commun. 47, 259 (1983).

[21] M. C. Bartelt, J. W. Evans, and M. L. Glasser, J. Chem. Phys. 99, 1438 (1993).

[22] M. F. Sykes and J. W. Essam, J. Math. Phys. 5, 1117 (1964).

[23] C. R. Scullard and J. L. Jacobsen, Phys. Rev. Res. 2, 012050 (2020).

[24] R. M. Ziff and C. R. Scullard, J. Phys. A: Math. Gen. 39, 15083 (2006)

[25] L. S. Ramirez, P. M. Centres, and A. J. Ramirez-Pastor, Phys. Rev. E 100, 032105 (2019).

[26] M. G. Slutskii, L. Y. Barash, and Y. Y. Tarasevich, Phys. Rev. E 98, 062130 (2018).
[27] S. Mertens and C. Moore, Phys. Rev. E 98, 022120 (2018).

[28] V. A. Cherkasova, Y. Y. Tarasevich, N. I. Lebovka, and N. V. Vygornitskii, Eur. Phys. J. B 74, 205 (2010).

[29] V. Cornette, A. J. Ramirez-Pastor, and F. Nieto, Eur. Phys. J. B 36, 391 (2003).

[30] V. Cornette, A. J. Ramirez-Pastor, and F. Nieto, Physica A 327, 71 (2003).

[31] Y. Leroyer and E. Pommiers, Phys. Rev. B 50, 2795 (1994).

[32] Y. Y. Tarasevich, N. I. Lebovka, and V. V. Laptev, Phys. Rev. E 86, 061116 (2012)

[33] Y. Y. Tarasevich, V. V. Laptev, N. V. Vygornitskii, and N. I. Lebovka, Phys. Rev. E 91, 012109 (2015).

[34] G. Kondrat, Z. Koza, and P. Brzeski, Phys. Rev. E 96, 022154 (2017).

[35] V. Cornette, A. Ramirez-Pastor, and F. Nieto, Phys. Lett. A 353, 452 (2006).

[36] N. I. Lebovka, Y. Y. Tarasevich, D. O. Dubinin, V. V. Laptev, and N. V. Vygornitskii, Phys. Rev. E 92, 062116 (2015).

[37] J. Krawczyk, T. Prellberg, A. L. Owczarek, and A. Rechnitzer, Phys. Rev. Lett. 96, 240603 (2006).

[38] P. Serra and J. F. Stilck, J. Phys. A: Math. Gen. 23, 5351 (1990).

[39] T. J. Oliveira, J. F. Stilck, and P. Serra, Phys. Rev. E 77, 041103 (2008).

[40] P. J. Flory, J. Am. Chem. Soc. 61, 1518 (1939).

[41] M. E. J. Newman and R. M. Ziff, Phys. Rev. Lett. 85, 4104 (2000).

[42] M. E. J. Newman and R. M. Ziff, Phys. Rev. E 64, 016706 (2001)

[43] H. Jang and U. Yu, Physica A 527, 121139 (2019).

[44] H. Tasaki, Commun. Math. Phys. 113, 49 (1987).

[45] A. B. Harris, J. Phys. C: Solid State Phys. 7, 1671 (1974).

[46] A. N. Berker, Physica A 194, 72 (1993).

[47] R. S. Nord and J. W. Evans, J. Chem. Phys. 82, 2795 (1985).

[48] M. J. de Oliveira, T. Tomé, and R. Dickman, Phys. Rev. A 46, 6294 (1992).

[49] E. J. Perino, D. A. Matoz-Fernandez, P. M. Pasinetti, and A. J. Ramirez-Pastor, J. Stat. Mech. (2017) 073206. 